\title{
The Classification of Physeal Injuries
}

\author{
N. K. Sferopoulos \\ From the Department of Pediatric Orthopaedics, "G. Gennimatas" Hospital, Thessaloniki, Greece \\ sferopoulos@in.gr
}

Conflict of interest statement: The author certifies that he has no commercial associations (such as consultancies, stock ownership, equity interest, patent/licensing arrangements, etc) that might pose a conflict of interest in connection with the submitted article. The author received no financial support for this study.

\begin{abstract}
The classification of physeal fractures in children is an important aspect for research and practice. The evaluation of a new, more capable classification system to classify all pediatric physeal fractures, than the current classification systems, is a work in progress. A review of the literature is performed focusing on the radiographically occult lesions of the Salter and Harris classification system, and on the recent publications suggesting new classification schemes.
\end{abstract}

\section{REVIEW}

The growth plate, also known as epiphyseal/physeal plate or physis, is weaker than the nearby tendons, ligaments or joint capsule, and obviously weaker than bone. Hence, ligamentous injuries, as well as traumatic dislocation of major joints are less common in childhood. However, fractures through bone are much more common in children. This, apparent paradox, may probably be explained by the fact that only shearing, splitting and avulsion forces are capable of separating a physeal plate. Hippocrates reported the first medical documentation of a physeal injury. The distal part of the radius is the most common site of physeal plate injury, if finger phalangeal physeal injuries are excluded [15].

Multiple classification systems of physeal fractures have been described. The most commonly used classification for pediatric physeal fractures was proposed by Salter and Harris in 1963 [1]. Their report, which includes both general and specific physeal plate injuries, distinguishes injuries of the epiphysis from physeal plate injuries. It also indicates three main types of a physeal plate injury, which include the separation of the epiphysis through the physeal plate, fractures that cross the physeal plate, and crushing injuries of the plate itself. The classification of physeal plate injuries in 5 types, proposed in their report, is related to the mechanism of injury, the relationship of the fracture line to the various cellular layers of the physeal plate, and the prognosis concerning disturbance of growth. Diagnosis of a physeal plate injury is based on the radiographic detection of the fracture line, but should be suspected in any child showing clinical evidence of fracture, dislocation, ligamentous rupture, or even severe sprain of a joint, that is radiographically occult.

Type $\mathrm{V}$ injury results from a severe crushing force applied through the epiphysis to an area of the physeal plate [1]. The existence of type $\mathrm{V}$ physeal injury has been questioned, since no cases fulfilling the diagnostic criteria were identified [6], but the idea that the concept should be removed from the classification scheme [7], or changed to include all Salter-Harris types I to IV injuries complicated by growth disturbance [8] was not favoured [9]. The primary etiological factor of a type V lesion was also thought to be ischemic damage rather than direct cellular compression in the germinal zone [6]. Histologic findings of the distal radius and ulna supported this concept suggesting that microscopic trauma may lead to microbridges, in the form of transphyseal linear ossific striations, which may not necessarily lead to complete arrest. However, they may interfere with growth leading to the well recognized variations in the relative length of the distal radius and ulna [10-12].

The efficacy of radiography and magnetic resonance imaging (MRI) to delineate a type V lesion was evaluated in two patients [13]. In the former patient, diagnosis was based on conventional radiographs, which indicated a severe impaction injury of the proximal tibia with focal diminution of the width of the physeal plate, but with no cortical fracture. In the latter patient, a nonradiographically 


\section{N. K. Sferopoulos}

evident injury was indicated by MRI. It revealed two circumscribed bone contusions opposing one another on either side of the medial femoral physeal plate. This finding was considered to be diagnostic of a Salter-Harris type V injury.

The radiographic diagnosis in the former patient was against the first of the three suggested inclusion criteria to diagnose a type $\mathrm{V}$ lesion.

The criteria include: (a) normal findings on roentgenograms taken in at least two planes at the time of injury, (b) no treatment, and (c) subsequent positive roentgenographic findings showing partial or complete closure of the physis [6]. However, it may be reasonable to consider that a severe impaction force on a growing long bone may cause crushing not only of the germinal cell layer, but may also cause focal trabecular damage and haemorrhage involving the adjacent epiphysis and metaphysis. Therefore, it may be mandatory to reconsider the first criterion. Imaging studies, including radiographs, computed tomography (CT) and MRI, may indicate a severe impaction injury associated with a diminished width of the growth plate, but with no fracture line. In addition, MRI using fatsuppression techniques may clearly indicate bone areas of high signal intensity, representing focal oedema and haemorrhage, following a microtrabecular injury of the bone marrow [14]. The appearance of focal bone bruising on both sides of the injured physeal plate, associated with local diminished width, may be considered pathognomonic of a Salter-Harris type V lesion.

Detailed imaging and histological techniques were used to assess the acute chondro-osseous injury in the child. They revealed two occult lesions: the incomplete Salter-Harris type I fracture and bone bruising [15-17].

The value of MRI has been evaluated to diagnose non-radiographically evident injuries of the distal forearm and wrist in 20 children, whose symptoms did not resolve after 5 weeks of immobilisation [18]. The MRI findings indicated the occult lesion of bone bruising in all cases. Three types of bone bruising involving the distal radius were identified. In type 1 injuries, bone bruising involved the metaphysis in close proximity to the distal radial growth plate. In type 2 injuries, bone bruising extended to both, the metaphysis and diaphysis. In type 3 injuries, bone bruising affected both sides of the distal radial growth plate. Type 1 injuries were consistent with complete, un-displaced Salter-Harris type I fractures. In type 3 lesions, the distribution of the bone marrow oedema on both sides of the growth plate was carefully estimated in order to diagnose potential Salter-Harris type V injuries. It was concluded that the most challenging aspects of MRI-based diagnosis of occult lesions of the distal radius is the identification of a bone bruise and the recognition of the very rare undisplaced Salter-Harris type I and type V injuries.

A 9-year-old girl with an occult, on plain radiography, injury of the distal femur was examined with MRI [19]. The findings included widening of the physeal plate, perichondrial disruption, intracartilaginous fracture, and adjacent metaphyseal oedema indicating a Salter-Harris type I fracture without displacement. Physeal widening may be evident on radiographs around the knee and in the distal tibial physeal plate 3-4 weeks after injury. This is a very rare radiographic finding following Salter-Harris type I, II or III injuries and may be considered as a significant factor heralding disturbance of growth [20].

Among the most suitable classification schemes of the injuries to the growth mechanism of the immature skeleton are those proposed by Ogden and Peterson, which represent a considerable expansion of the Salter-Harris classification. Ogden created more types and subtypes of injury patterns presenting a more extensive classification scheme, while Peterson described two new types of physeal fractures [10, 21]. The use of both classification schemes has been proved to be of significant diagnostic and clinical importance [21,22]. The value of both classifications systems was recently examined to evaluate whether they were sufficient to include all physeal fractures of the distal radius that could not be included in the Salter-Harris classification system [23]. Physeal injuries included fractures through the physeal plate, as well as fractures extending to or crossing the physeal plate. From a total number of 292 children with a physeal fracture that could not be classified according to the Salter-Harris system, 96 injuries could not fit to either of the classification systems proposed by Ogden and Peterson. Five types of physeal fractures, which could not be classified with the SalterHarris system, were described. In type 1 injuries, a transverse fracture of the diaphysis or metaphysis, which involved the whole width of the cortex of the distal radius and was associated with one or more longitudinal linear fracture lines extending to the physeal plate, was evident. Displacement on type 1 


\section{The Classification of Physeal Injuries}

injuries was seen only at the level of the transverse diaphyseal/metaphyseal fracture of the radius and was never evident within the distal fragment or at the level of the physeal plate. In type 2 injuries, there was a transverse physeal fracture associated with a usually large metaphyseal fragment, which was subdivided by one or more fracture lines that also extended to the physeal plate. Displacement on type 2 injuries was seen at the level of the physeal fracture but was never evident within the metaphyseal fragment of the radius. In type 3 injuries, there was a physeal fracture associated with two metaphyseal fragments of the distal radial metaphysis: one of the fragments was always separated from the epiphysis and the other one was attached to the epiphysis. Type 4 injuries may include any type of physeal injury associated with a fracture line extending beyond the physeal plate to the epiphysis, which is not described in the Salter-Harris scheme. Finally, the classification of any of the previous types complicated by asymmetric growth or premature physeal arrest was changed to a type 5 lesion. The proposed modified scheme incorporates all previous classification systems, allows classification of all physeal injuries of the distal radius that are not included in the Salter-Harris system and may assist guiding and evaluating management and treatment outcomes for the whole spectrum of these injuries.

Diagnosis of the types 1 to 4 fractures, not included in the Salter-Harris classification, may routinely be based on the radiographic examination (Fig. 1, 2, 3). However, a limited number of cases may necessitate evaluation using CT or MRI (Fig. 4). Treatment modalities should always focus on the principles of treatment proposed by Salter and Harris. They refer to the gentleness of reduction, the time of reduction, the method of reduction, the acceptable position of reduction and the period of immobilization [1]. This is the only secure way to avoid further damage of a disrupted physeal plate and diminish the incidence of partial or complete premature physeal arrest.

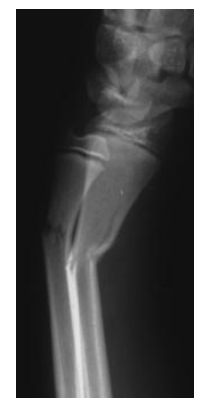

Figure1. Lateral Radiograph of a 12-Year-Old Boy Indicated an Angulated Type 1 Fracture of the Radius

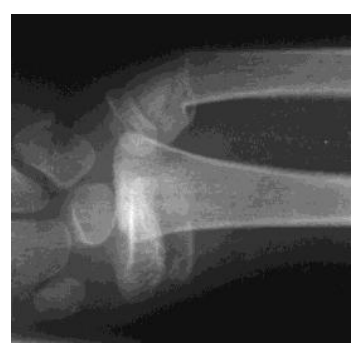

Figure2. Anteroposterior Radiograph of a 10-Year-Old Boy Indicated a Displaced Type 2 Fracture of the Radius

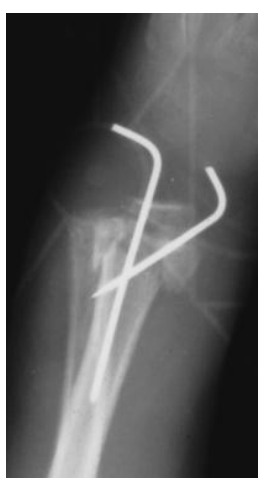

Figure3. Lateral Radiograph of an 8-Year-Old Boy with a Displaced Type 3 Fracture of the Radius Following Surgical Treatment 


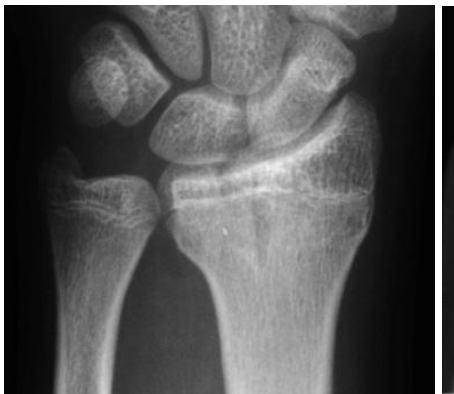

(a)

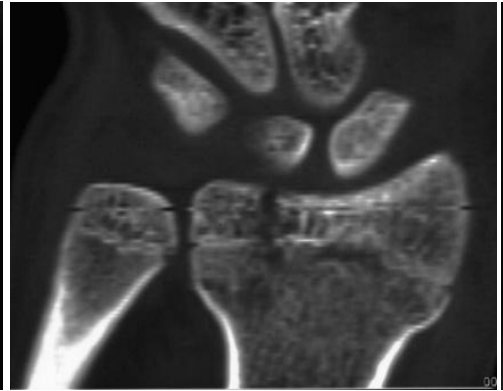

(b)

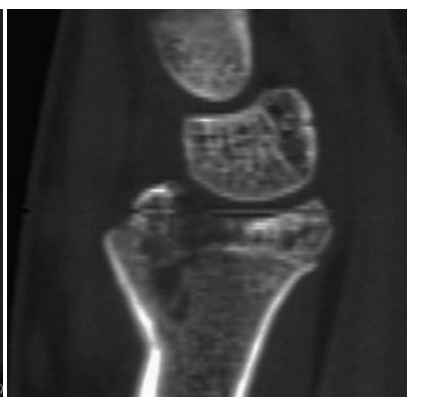

(c)

Figure4. Anteroposterior Radiograph (a) of a 15-Year-Old Boy Indicated a Radial Fracture, which Could not be Classified Using the Salter-Harris Scheme. Intraepiphyseal Extension of the Fracture Line that was Shown on the Anteroposterior (b) and Lateral (c) CT Views Secured the Diagnosis of a Type 4 Lesion of the Radius

\section{REFERENCES}

[1] Salter R.B., Harris W.R. Injuries involving the epiphyseal plate, J. Bone Joint Surg. Am. 1963; 45(3): 587-622.

[2] Mizuta T., Benson W.M., Foster B.K., Paterson D.C., Morris L.L. Statistical analysis of the incidence of physeal injuries, J. Pediatr. Orthop. 1987;7(5):518-523.

[3] Mann D.C., Rajmaira S. Distribution of physeal and nonphyseal fractures in 2,650 longbone fractures in children aged 0-16 years, J. Pediatr. Orthop. 1990;10(6):713-716.

[4] Peterson H.A. Epiphyseal growth plate fractures. Berlin Heidelberg: Springer-Verlag, 2007.

[5] Jaramillo D., Shapiro F. Musculoskeletal trauma in children. Magn.Reson.Imaging Clin. N.Am. 1998; 6(3):521-536.

[6] Peterson H.A., Burkhart S.S. Compression injury of the epiphyseal growth plate: fact or fiction? J. Pediatr. Orthop. 1981;1(4):377-384.

[7] Rang M. Injuries of the epiphyses, the growth plate, and the perichondral ring. In: Children's fractures. 2nd ed. Philadelphia: JB Lippincote, 1983, pp 19.

[8] Letts R.M. Compression injuries of the growth plate. In: Uhthoff HK, Wiley JJ (eds). Behavior of the Growth Plate. Raven Press, New York, 1988, pp 111-118.

[9] Peterson H.A. Premature closure of the distal radial physis after fracture of the distal radial metaphysis, J. Pediatr. Orthop. 1997;17(1):126-127.

[10] Ogden J.A. Injury to the growth mechanisms of the immature skeleton, Skeletal Radiol. 1981; 6(4): 237-253.

[11] Ogden J.A. Transphyseal linear ossific striations of the distal radius and ulna, Skeletal Radiol. 1990;19(3):173-180.

[12] Kennon J.C., Ganey T.M., Gaston R.G., Ogden J.A. Continued growth after limited physeal bridging, J. Pediatr. Orthop. 2013; 33 (8) : 857-861.

doi:10.1097/BPO.0b013e31829c008b.

[13] Sferopoulos N.K. Type V physeal injury, J. Trauma. 2007;63(6):E121-123.

[14] Meyers S.P., Wiener S.N. Magnetic resonance imaging features of fractures using the short tau inversion recovery (STIR) sequence: correlation with radiographic findings, Skeletal Radiol. 1991;20(7):499-507.

[15] Ogden J.A., Ganey T., Light T.R., Southwick W.O. The pathology of acute chondroosseous injury in the child, Yale J. Biol. Med. 1993;66(3):219-233.

[16] Laor T., Hartman A.L., Jaramillo D. Local physeal widening on MR imaging: an incidental finding suggesting prior metaphyseal insult, Pediatr. Radiol. 1997; 27(8):654662. 
[17] Ogden J.A. Skeletal injury in the child. New York, Springer; 2000.

[18] Sferopoulos N.K. Bone bruising of the distal forearm and wrist in children, Injury. 2009;40(6):631-7. doi: 10.1016/j.injury.2009.01.104. Epub 2009 Apr 24.

[19] Chauvin N., Jaramillo D. Occult distal femoral physeal injury with disruption of the perichondrium, J. Comput. Assist. Tomogr. 2012; 36(3):310-312. doi: 10.1097/RCT.0b013e31825039a6.

[20] Sferopoulos N.K. Concomitant physeal fractures of the distal femur and proximal tibia, Skeletal Radiol. 2005; 34(7):427-430. Epub 2005 Feb 16.

[21] Peterson H.A. Physeal fractures: Part 3. Classification, J. Pediatr. Orthop. 1994; 14(4): 439-448.

[22] Cannata G., De Maio F., Mancini F., Ippolito E. Physeal fractures of the distal radius and ulna: long-term prognosis, J. Orthop. Trauma. 2003; 17(3): 172-179.

[23] Sferopoulos N.K. Classification of distal radius physeal fractures not included in the Salter-Harris system, Open Orthop. J. 2014; 11;8:219-224.

doi: 10.2174/ 187432500140 8010219. eCollection 2014. 